\title{
Deciphering Low-Code/No-Code Hype - Study of Trends, Overview of Platforms, and Rapid Application Development Suitability
}

\author{
Mayuresh Kulkarni* \\ * Master of Engineering Management, Case Western Reserve University \\ *Bachelor of Engineering, University of Mumbai \\ DOI: 10.29322/IJSRP.11.07.2021.p11570 \\ http://dx.doi.org/10.29322/IJSRP.11.07.2021.p11570
}

\begin{abstract}
Low Code and No Code (LCNC) are platforms that allow for the development of software application with little to no coding. These platforms are latest on the block and are surrounded by a large amount of buzz. This paper analyses the LCNC trends, overview of common platform features, and its suitability for Rapid Application Development (RAD).
\end{abstract}

Index Terms- Low-Code, No Code, LCNC, System Implementation, IT Process Management, Architecture and Technology Strategy, Digital Transformation, Leading IT Trends, Packaged Solution, Modern Platforms, Citizen Developer Tools, Automation, Rapid Application Development, Rapid Prototyping.

\section{INTRODUCTION}

$\mathrm{D}$ igital strategy is at the core of every organization's growth and customer experience journey. This strategy is enabled by developing and timely delivering software applications to support the digital vision. Most businesses have complex and evolving software application needs that are historically supported by the organization's Information Technology (IT) departments. The reliance on IT has been due to their technical expertise which also meant that organizations have to compete internally for a narrow pool of resources. Even if the IT resources are secured, the traditional Waterfall based Software Development Life Cycle (SDLC) approach involves long cycle of requirements gathering, application design, development, and UAT before being deployed to the end user. IT resource crunch along with a long SDLC has been the Achilles heel for the organizations to innovate at speed.

In early 2000, the Agile Manifesto [2] was created by a group of 17 industry thought leaders which supported Rapid Application Development [2] by shifting away from Waterfall to Scrum/Agile using iterative SDLC concepts. Even with this shift in SDLC, the reliance on IT within an organization has been persistent. LCNC has the potential to reduce this dependency by providing an integrated software development environment with a user-friendly interface that supports rapid application development. The degree of "coding" for developing applications on these platforms depend on the type of vendor selected and the baseline expectation is support for dragging and dropping configuration widgets and UI components. As a result, non-IT resources also referred as "citizen developers" [3] are able to learn these LCNC platforms for developing applications in conjunction or independently of the organization's IT, thereby reducing the tactical reliance on IT with an estimate of making application development to be 10 times faster [4] than traditional code-based development. This is an evolving space already being flooded with a large list of vendors with key themes, trends, platform features, and insights on RAD suitability starting to emerge.

\section{KeY LCNC TRENDS AND THEMES}

As part of LCNC trends, the current global LCNC market and its outlook is assessed to validate if the hype truly holds true. Additionally, a select pool of vendors is narrowed down to evaluate them against each other.

\section{A. Market Growth Indicator}

Per the 2020 Grandview Research [5], LCNC market is expected to grow at a CAGR of $22.7 \%$ form 2020 to 2027 and cross \$21B in 2021. By the year 2027, this is predicted to be a $\$ 86.9 \mathrm{~B}$ industry with close to even split between On Premise and Cloud based LCNC platforms.

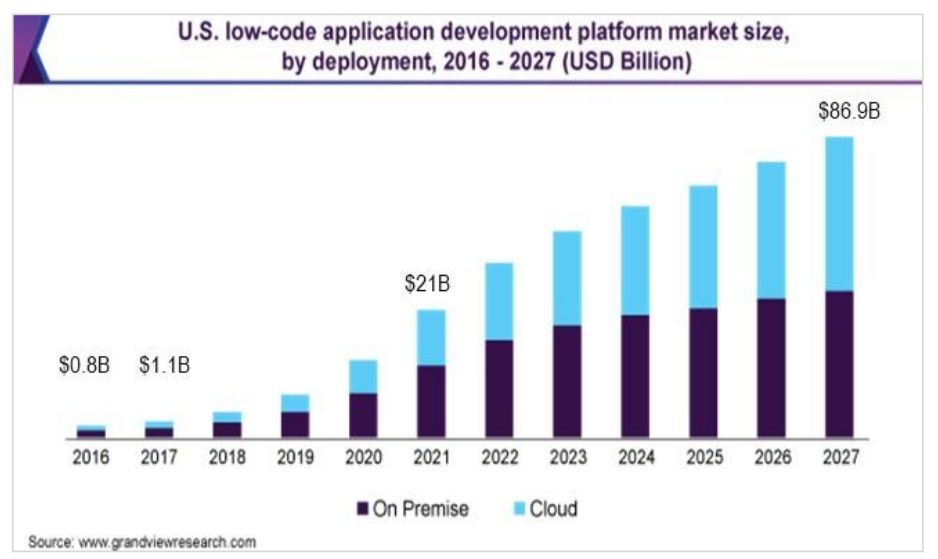

Fig. 1 Projected Low-Code Development Platform Market Growth. 


\section{B. Comparison of LCNC Growth with Other Leading Technology Trends}

For the purposes of this analysis, LCNC growth is compared against AI, Blockchain, Edge Computing, and RPA. Also, to ensure research consistency, the analyses refer market reports from a common vendor. [5],[6],[7],[8],[9].

* Market Size for 2027

LCNC Global Market Size Vs. Other Leading Trends

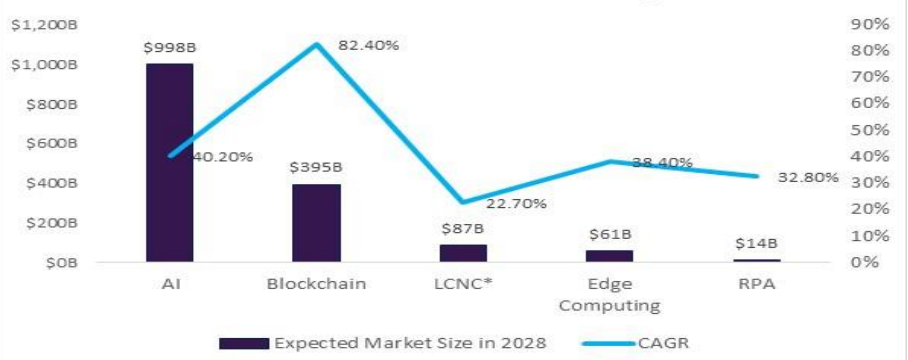

Fig. 2 LCNC Global Market Size Vs. Other Leading Trends.

While the growth for LCNC has been unprecedented, it fairs moderately when stacked against other leading technology trends. In fact, the CAGR for LCNC is the lowest at $22.7 \%$ when compared to others signifying interplay with other trends e.g., AI and RPA platforms potentially chipping away some of the LCNC market cap.

\section{Leading LCNC Platforms Based on Analyst Reports}

Over 300 vendors are estimated [10] to cater the LCNC market. While it is not possible to cover all of them, some of the vendors repeatedly rise above the others in the pack across the 3 analyst reports. The below table illustrates these select leading LCNC vendors [5], [11], [12].

\begin{tabular}{|l|c|c|c|}
\hline LCNC Vendors & Forrester's Report & Gartner's Report & Grand View's Report \\
\hline Appian & Strong Performer & Leader & Prominent Player \\
\hline Mendix & Leader & Leader & Prominent Player \\
\hline Microsoft Power Apps & Leader & Leader & Prominent Player \\
\hline OutSystems & Leader & Leader & Prominent Player \\
\hline Pegasystems & Strong Performer & Visionary & Prominent Player \\
\hline Salesforce & Strong Performer & Leader & Prominent Player \\
\hline ServiceNow & Leader & Leader & Prominent Player \\
\hline
\end{tabular}

Table. 1 Leading LCNC Platforms.

\section{Funding \& Market Capitalization of Leading LCNC Vendors}

At least $\$ 800 \mathrm{M}+$ is raised by group of vendors listed below. [13], [14], [15], 16], [17]

\begin{tabular}{|l|c|c|}
\hline LCNC Vendors & Funding Raised & Market Cap \\
\hline Appian & $\$ 48 \mathrm{M}$ & $\$ 9.5 \mathrm{~B}$ \\
\hline Mendix & $\$ 38 \mathrm{M}$ & $\$ 0.7 \mathrm{~B}$ \\
\hline Microsoft Power Apps & $\mathrm{N} / \mathrm{A}$ & $\mathrm{N} / \mathrm{A}$ \\
\hline OutSystems & $\$ 572 \mathrm{M}$ & $\$ 9.5 \mathrm{~B}$ \\
\hline Pegasystems & $\mathrm{N} / \mathrm{A}$ & $\$ 11.0 \mathrm{~B}$ \\
\hline Salesforce & $\$ 65 \mathrm{M}$ & $\$ 230.0 \mathrm{~B}$ \\
\hline ServiceNow & $\$ 84 \mathrm{M}$ & $\$ 110.9 \mathrm{~B}$ \\
\hline \multirow{2}{*}{} & $\mathbf{\$ 8 0 7 M}$ & $\$ \mathbf{M 6 1 B}$ \\
\hline
\end{tabular}

Table. 2 Funding and Market Capitalization of Leading LCNC Platforms.

This publication is licensed under Creative Commons Attribution CC BY.

http://dx.doi.org/10.29322/IJSRP.11.07.2021.p11570
Market Cap is based on the stock price on July 7th, 2021 for publicly traded firms. Since Mendix and OutSystems are not traded, valuation is used for comparison purposes [18], [19]. The total valuation of this pool of vendors exceeds $\$ 361 \mathrm{~B}$. This number will be significantly higher when factored for the 300 vendors estimated [10] to cater this segment and will significantly flood the market. Even with a medium to high CAGR of $22 \%$ (Fig 2), LCNC platforms will need to stay laser focused on their product and market execution strategy to avoid losing on growth and staying relevant.

\section{E. Industry Demographics of the Leading LCNC Vendors}

Conceptually, LCNC can be industry agnostic however some industries have a higher propensity for being able to leverage these platforms due to existing legacy technologies that need to be wrapped around LCNC for creating new application or their higher appetite for innovation. This combined with sales and marketing focus of LCNC vendors paint the picture for their industry alignment. The below table is indicative and based on the population of reviews provided for these vendors by their customers. [21], [22], [23], [24], [25], [26].

\begin{tabular}{|c|c|c|c|c|c|}
\cline { 2 - 6 } \multicolumn{1}{c|}{} & \multicolumn{5}{c|}{ Industry Demographics } \\
\cline { 2 - 6 } \multicolumn{1}{c|}{} & Services & $\begin{array}{c}\text { Financial } \\
\text { Services }\end{array}$ & Manufacturing & Healthcare & Other \\
\hline Appian & $16 \%$ & $40 \%$ & $7 \%$ & $6 \%$ & $31 \%$ \\
\hline Mendix & $32 \%$ & $17 \%$ & $20 \%$ & $2 \%$ & $29 \%$ \\
\hline Microsoft & $20 \%$ & $12 \%$ & $20 \%$ & $5 \%$ & $43 \%$ \\
\hline OutSystems & $27 \%$ & $21 \%$ & $10 \%$ & $7 \%$ & $35 \%$ \\
\hline Pegasystems & N/A & N/A & N/A & N/A & N/A \\
\hline Salesforce & $30 \%$ & $15 \%$ & $19 \%$ & $8 \%$ & $28 \%$ \\
\hline ServiceNow & $25 \%$ & $17 \%$ & $12 \%$ & $12 \%$ & $34 \%$ \\
\hline
\end{tabular}

Indicates vendor with highest demographic focus

Table. 3 Industry Demographics of Leading LCNC Platforms.

\begin{tabular}{|c|c|c|c|c|}
\cline { 2 - 5 } \multicolumn{1}{c|}{} & \multicolumn{4}{c|}{ Geographic Demographics } \\
\cline { 2 - 5 } \multicolumn{1}{c|}{} & North America & $\begin{array}{c}\text { Europe, Middle } \\
\text { East, \& Africa }\end{array}$ & Asia/Pacific & Latin America \\
\hline Appian & $49 \%$ & $31 \%$ & $16 \%$ & $4 \%$ \\
\hline Mendix & $28 \%$ & $63 \%$ & $7 \%$ & $2 \%$ \\
\hline Microsoft & $37 \%$ & $28 \%$ & $26 \%$ & $9 \%$ \\
\hline OutSystems & $28 \%$ & $50 \%$ & $16 \%$ & $6 \%$ \\
\hline Pegasystems & N/A & N/A & N/A & N/A \\
\hline Salesforce & $52 \%$ & $24 \%$ & $16 \%$ & $8 \%$ \\
\hline ServiceNow & $49 \%$ & $18 \%$ & $25 \%$ & $8 \%$ \\
\hline
\end{tabular}

Indicates vendor with high demographic focus

Table. 4 Geographic Demographics of Leading LCNC Platforms.

Services, Finance, and Manufacturing are three primary sectors providing traction to LCNC based on Table 3. From a geographic standpoint and referring to Table 4, Appian, Salesforce, and ServiceNow command the North American Market and are USA headquartered. OutSystems originated in EMEA which led to their stronghold in this region. Microsoft on the other hand has more of a distributed hold across both the industry and geographic demographics. The above tables are indicative and based on the population of reviews provide for these vendors by their customers. [21], [22], [23], [24], [25], [26]. 


\section{LCNC PLATFORM OVERVIEW}

\section{A. Key LCNC Platform Features}

There are few common native core features that most LCNC platforms support. The maturity levels of these features do vary based on the platform selected. Below is the high-level overview of these:

1) Developer studio or workbench - An Integrated Development Environment (IDE) that allows developers to interact with the LCNC platform to build applications.

2) UI components and widgets - UI screen elements and widgets for visualization that can be dragged and dropped along with ability to modify HTML and CSS if needed.

3) Data integration - Tools for integrating, transposing, and orchestrating the exchange with internal and external data sets.

4) Workflows and rules - Configurable workflow and rules that allows for application to hand off with either other parts of the application or amongst application users.

5) Process automation - Bots and Robotic Process Automation capabilities for assisting with manual and repeatable processes

6) Testing and deployment management - Tools for application administration, unit, functional, and integration testing, and deployment.

7) Application governance - Super admin user tools for rolesbased access management, security settings, privacy management, data oversight, performance monitoring, audit logging, and reporting.

\section{B. LCNC Representative Technology Components and Stack}

Features needed to enable the LCNC platform are supported by an array of technology stacks. These are often a combination of open source and licensed technologies which vary from vendor to vendor.[27], [28], [29].

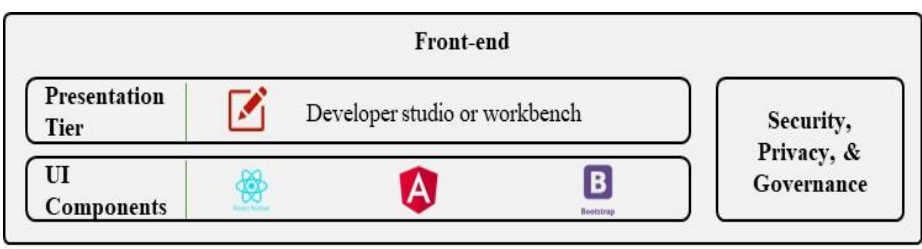

\begin{tabular}{|c|c|c|c|c|}
\hline \multicolumn{4}{|c|}{ Middle and Back-end } & \\
\hline $\begin{array}{l}\text { Webserver } \\
\text { \& API Tier } \\
\end{array}$ & node & * HAPROXY & (M) Mulesoft & \multirow{6}{*}{$\begin{array}{c}\text { Security, } \\
\text { Privacy, \& } \\
\text { Governance }\end{array}$} \\
\hline $\begin{array}{l}\text { Workflow \& } \\
\text { Rules Tier }\end{array}$ & : kissflow & ODrools & Spring cloud & \\
\hline $\begin{array}{l}\text { Automation } \\
\text { Tier }\end{array}$ & $\bigoplus$ ChatBot & Ui] Path & ARGOS $L A B S$ & \\
\hline $\begin{array}{l}\text { Database } \\
\text { Tier } \\
\end{array}$ & (6) Possuresol. & mongoDB. & \$lgnite & \\
\hline $\begin{array}{l}\text { Orchestration } \\
\text { Tier } \\
\end{array}$ & docker & FANCHER & kubernetes & \\
\hline $\begin{array}{l}\text { Infrastructure } \\
\text { Tire }\end{array}$ & $\Delta_{\text {Auve }}^{\text {Micosoft }}$ & 2 & 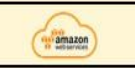 & \\
\hline
\end{tabular}

Fig. 3 LCNC Representative Technology Components and Stack.

\author{
IV. LCNC AND ITS SUITABILITY FOR \\ RAPID APPLICATION DEVELOPMENT (RAD)
}

\section{A. LCNC Synergies with RAD}

The term Rapid Application Development (RAD) [2] was coined by James Martin in his book Rapid Application Development (RAD). This is an application development lifecycle designed to give much faster development and higher-quality results than those achieved with the traditional lifecycle. The advent of LCNC platforms enable this type of modern application development methodology due to complementary synergies mainly due to following reasons:

1) Learning curve to start building applications is compressed and typically in weeks.

2) LCNC platforms provide an integrated environment to the user that encompasses all the needed technology stacks, which the developer does not need to manage and maintain.

3) User story to application build and deployment can be done by a lean self-sufficient team with limited technical expertise vs. heavy reliance on IT.

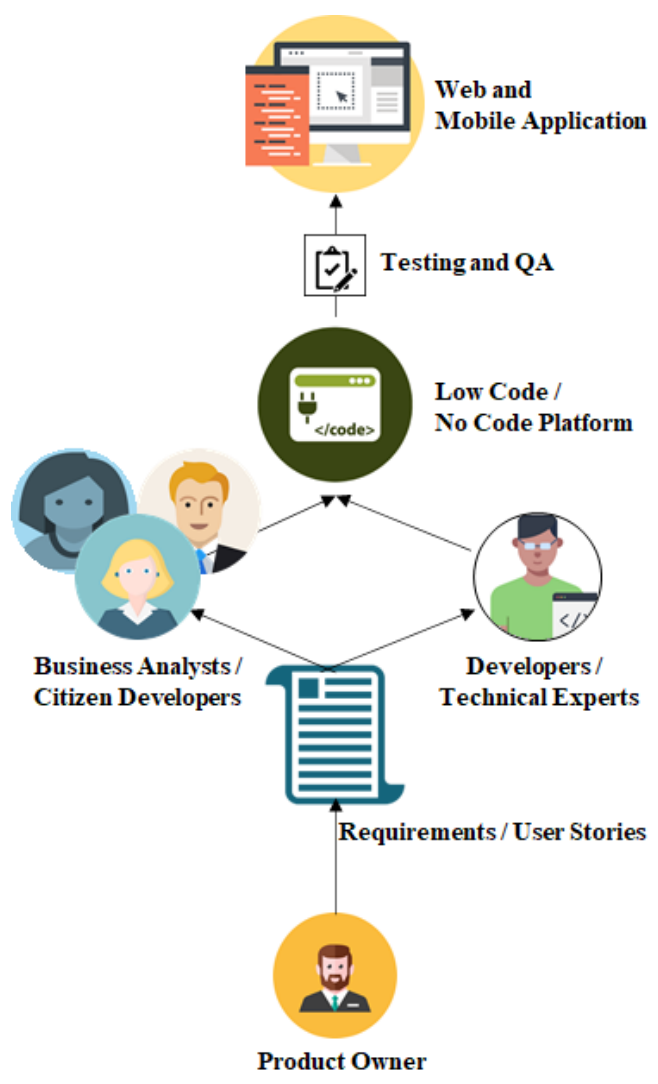

Fig. 4 Lean and Self-Sufficient LCNC Development Team.

4) Hybrid agile style iterations [30] to develop the application with certainty and specified timeline is possible due to reduced resource constrains as citizen developers shoulder the heavy lifting while doing so in a shorter build, test, and deployment cycle. 


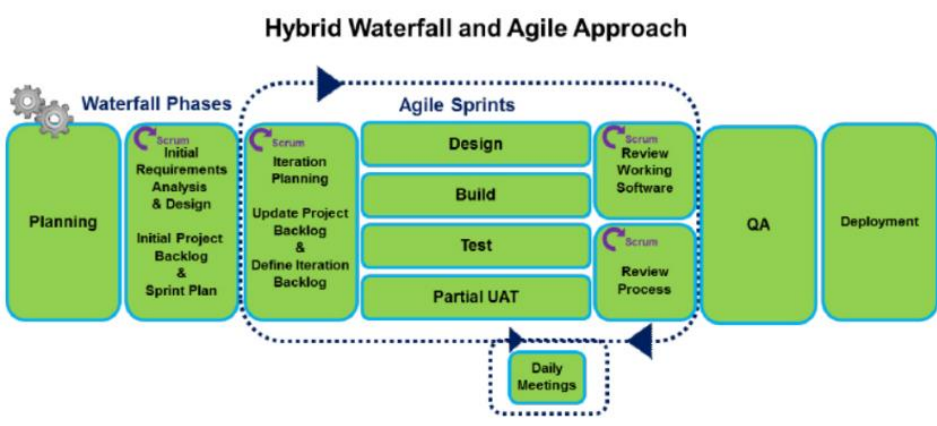

Fig. 5 Hybrid Agile Approach for LCNC Development.

\section{B. LCNC Program Blind Spots to Consider While in RAD}

While there is strong market demand for LCNC platforms and a great synergy for RAD, there are few blind spots that every LCNC program will need to navigate. These are:

1) Ensuring the platform fits the objectives and provides scale. Given the over 300 vendors [10] in this space, it is necessary that appropriate vendor selection is done so avoid future regrets.

2) Not compromising on the security of applications built using LCNC. This becomes increasingly critical during RAD as the focus is on building applications vs. making them secure and safe.

\section{CONCLUSION}

By 2024, 75\% of large enterprises will be using at least four lowcode development tools for both IT application development and citizen development initiatives [31]. This along with other trends discussed in this paper points to LCNC being more than a hype; it is here to stay and will be the new normal for application development. Also, business can successfully adopt these LCNC platforms for RAD with a hybrid Agile model proposed in this paper. In doing so, it is also necessary to understand the complex architecture that underline these platforms and plan for navigating some of the blind spots so the application design, delivery, and deployment is holistically successful.

\section{REFERENCES}

[1] Manifesto for Agile Software Development. (2021). Agile Manifesto. http://agilemanifesto.org/

[2] Martin, J. (1991). Rapid Application Development. Macmillan Coll Div.

[3] Definition of Citizen Developer - Gartner Information Technology Glossary. (n.d.). Gartner. Retrieved June 21, 2021, from https://www.gartner.com/en/information-technology/glossary/citizendeveloper\#:\%7E:text=A\%20citizen\%20developer\%20is\%20an,by\%20IT\% 20or\%20business\%20units.\&text=They\%20report $\% 20$ to $\% 20 \mathrm{a} \% 20 \mathrm{busines}$ s,citizen\%20developers\%20are\%20business\%20technologists

[4] Rymer, J. (2018, September 21). Why You Need To Know About LowCode, Even If You're Not Responsible For Software Delivery. Forrester. https://go.forrester.com/blogs/why-you-need-to-know-about-low-codeeven-if-youre-not-responsible-for-software-delivery/

[5] Low-Code Application Development Platform Market Report, 2020-2027. (2020). Grand View Research.

https://www.grandviewresearch.com/industry-analysis/low-codeapplication-development-platform-

market?utm_source=prnewswire\&utm_medium=referral\&utm_campaign= ict_02-sep-20\&utm_term=low-code-application-development-platformmarket\&utm_content=rd

[6] Artificial Intelligence Market Worth $\$ 997.77$ Billion By 2028. (n.d.). Grand View Research. Retrieved July 7, 2021, from https://www.grandviewresearch.com/press-release/global-artificialintelligence-ai-

market\#:\%7E:text=The\%20global\%20artificial\%20intelligence\%20market ,40.2\% 25\%20from\%202021\%20to\%202028.

[7] Blockchain Technology Market Worth $\$ 394.60$ Billion By 2028. (n.d.). Grand View Research. Retrieved July 7, 2021, from https://www.grandviewresearch.com/press-release/global-blockchaintechnology-market

[8] Edge Computing Market Size Worth \$61.14 Billion By 2028. (n.d.). Grand View Research. Retrieved July 7, 2021, from

https://www.grandviewresearch.com/press-release/global-edge-computingmarket

[9] Robotic Process Automation Market Worth \$13.74 Billion By 2028. (n.d.). Grand View Research. Retrieved July 7, 2021, from

https://www.grandviewresearch.com/press-release/global-robotic-processautomation-rpa-market

[10] Alexander, F., Alexander, F., Mates, N., \& Chillingworth, M. (2021, January). Top 5 Benefits of Low-Code. Outsystems. https://www.outsystems.com/blog/posts/benefits-of-low-code-platforms/

[11] The Forrester Wave ${ }^{\mathrm{TM}}$ : Low-Code Development Platforms For Professional Developers, Q2 2021. (n.d.). Forrester. Retrieved July 7 , 2021, from

https://www.forrester.com/report/The+Forrester+Wave+LowCode+Develo pment+Platforms+For+Professional+Developers+Q2+2021/-/ERES161668

[12] Gartner, Inc. (n.d.). Enterprise LCAP (Low-Code Application Platforms) Reviews 2021 | Gartner Peer Insights. Gartner. Retrieved July 7, 2021, from https://www.gartner.com/reviews/market/enterprise-low-codeapplication-platform

[13] Appian - Crunchbase Company Profile \& Funding. (n.d.). Crunchbase. Retrieved July 10, 2021, from

https://www.crunchbase.com/organization/appian

[14] Mendix - Crunchbase Company Profile \& Funding. (n.d.). Crunchbase. Retrieved July 10, 2021, from https://www.crunchbase.com/organization/mendix

[15] OutSystems - Crunchbase Company Profile \& Funding. (n.d.). Crunchbase. Retrieved July 10, 2021, from https://www.crunchbase.com/organization/outsystems

[16] Salesforce - Crunchbase Company Profile \& Funding. (n.d.). Crunchbase. Retrieved July 10, 2021, from https://www.crunchbase.com/organization/salesforce

[17] ServiceNow - Crunchbase Company Profile \& Funding. (n.d.). Crunchbase. Retrieved July 10, 2021, from https://www.crunchbase.com/organization/service-now-com

[18] Rymer, J. (2018a, September 13). Siemens Snaps Up Mendix; Low-Code Platforms Enter New Phase. Forrester.

https://go.forrester.com/blogs/siemens-snaps-up-mendix-low-codeplatforms-enter-new-phase/

[19] OutSystems, Software Development Platform Leader, Raises \$150 Million Investment at $\$ 9.5$ Billion Valuation. (n.d.). OutSystems. Retrieved July 7 , 2021, from https://www.outsystems.com/news/modern-applicationplatforminvestment/\#:\%7E:text=The\%20round $\% 2 \mathrm{C} \% 20 \mathrm{co} \% 2 \mathrm{Dled} \% 20 \mathrm{by}$, values $\%$ 20OutSystems\%20at\%20\%249.5\%20billion.

[20] Commissioned by Red Hat. (2018, February). Intelligent Process Automation and the Emergence of Digital Automation Platforms. 451 Research Advisory. https://www.redhat.com/cms/managed-files/mi-451research-intelligent-process-automation-analyst-paper-f11434-201802.pdf

[21] Gartner, Inc. (n.d.-a). Appian Reviews, Ratings, and Features - Gartner 2021. Gartner. Retrieved July 8, 2021, from https://www.gartner.com/reviews/market/enterprise-low-code-applicationplatform/vendor/appian

[22] Gartner, Inc. (n.d.-c). Mendix Reviews, Ratings, and Features - Gartner 2021. Gartner. Retrieved July 8, 2021, from https://www.gartner.com/reviews/market/enterprise-low-code-applicationplatform/vendor/mendix

[23] Gartner, Inc. (n.d.-d). Microsoft Reviews, Ratings, and Features - Gartner 2021. Gartner. Retrieved July 8, 2021, from

https://www.gartner.com/reviews/market/enterprise-low-code-applicationplatform/vendor/microsoft

[24] Gartner, Inc. (n.d.-e). OutSystems Reviews, Ratings, and Features Gartner 2021. Gartner. Retrieved July 8, 2021, from https://www.gartner.com/reviews/market/enterprise-low-code-applicationplatform/vendor/outsystems 
[25] Gartner, Inc. (n.d.-f). Salesforce Reviews, Ratings, and Features - Gartner 2021. Gartner. Retrieved July 8, 2021, from

https://www.gartner.com/reviews/market/enterprise-low-code-applicationplatform/vendor/salesforce

[26] Gartner, Inc. (n.d.-g). ServiceNow Reviews, Ratings, and Features -

Gartner 2021. Gartner. Retrieved July 8, 2021, from

https://www.gartner.com/reviews/market/enterprise-low-code-applicationplatform/vendor/servicenow

[27] Awan, S. (2018, June 17). Anatomy of Low-Code SaaS Development Platform - trillo-platform. Medium. https://medium.com/trillo-

platform/anatomy-of-low-code-saas-development-platform-d2e4b4ebd5ca

[28] Infrastructure architecture and deployment options. (2021, July 7).

OutSystems.

https://success.outsystems.com/Support/Enterprise_Customers/Infrastructu re_architecture_and_deployment_options?_gl=1*8ri36h*_ga*MTIzNzM4 MTMyNy4xNjI1MDY3MzQ3*_ga_ZD4DTMHWR2*MTYyNTc2MjUx NC4xMy4wLjE2MjU3NjI1MTQuNjA.

[29] Mendix. (2021, March 8). Enterprise Architecture Platform - Modules \& Services | Mendix Evaluation Guide. https://www.mendix.com/evaluationguide/enterprise-capabilities/platform-architecture/\#

[30] Archer, S. \& Kaufman, C. (2013). Accelerating outcomes with a hybrid approach within a waterfall environment. Paper presented at PMI® Global Congress 2013-North America, New Orleans, LA. Newtown Square, PA: Project Management Institute.

[31] Low-Code Development Technologies Evaluation Guide. (2019). Gartner. https://www.gartner.com/en/documents/3902331/low-code-developmenttechnologies-evaluation-guide

\section{AUTHOR}

Name - Mayuresh Kulkarni,

Master of Engineering Management,

Case Western Reserve University, Cleveland, Ohio, USA;

Bachelor of Engineering,

University of Mumbai, Mumbai, India;

mayuresh.c.k@gmail.com 